Current account

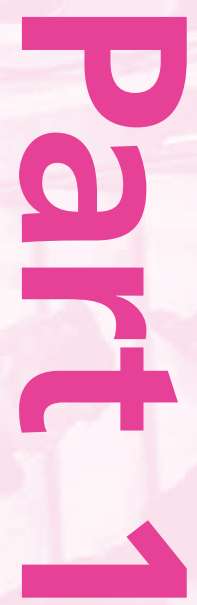




\section{Summary of balance of payments}




\section{Current account}

The UK has recorded a current account deficit in every year since 1984. Prior to 1984, the current account recorded a surplus in 1980 to 1983 . Since the last surplus was recorded in 1983, there have been four main phases in the development of the current account. In the first phase, from 1984 to 1989, the current account deficit increased steadily to reach a high of f25.5 billion in 1989, equivalent to -4.9 per cent of Gross Domestic Product (GDP). During the second phase, from 1990 until 1997, the current account deficit declined to a low of $f 1.0$ billion in 1997. In the third phase, between 1998 and 2006, the current account deficit widened sharply, peaking at $£ 44.9$ billion in 2006. This was the highest recorded in cash terms but only equated to -3.4 per cent of GDP. In the past two years there has been a reduction in the current account deficit - in 2009 it currently stands at $£ 15.5$ billion, equivalent to -1.1 per cent of GDP.

The profile for the current account has historically followed that of trade in goods, its biggest and most cyclical

\section{Figure 1.1}

\section{Current account balance}

f billion

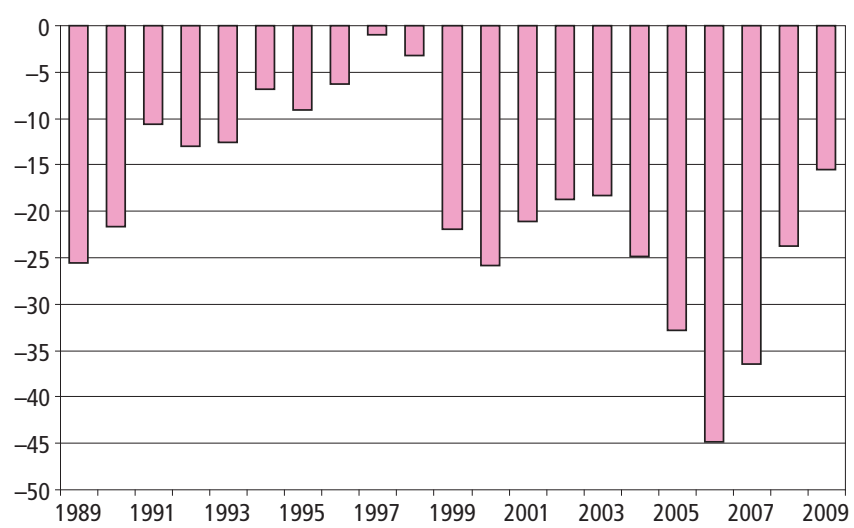

\section{Figure 1.2}

\section{Current balance as a percentage of GDP}

Per cent

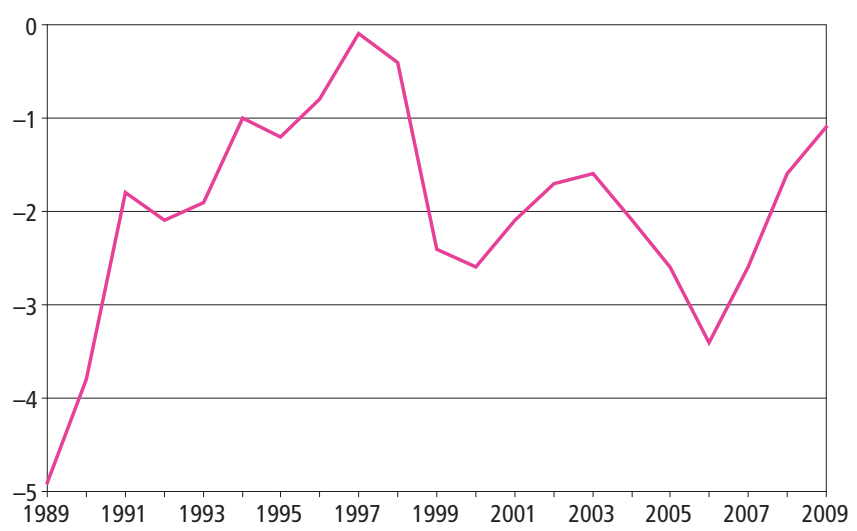

component. That pattern was broadly followed until it changed in 2001. The pattern re-emerged in 2004 to 2006 with an increasing deficit on trade in goods being mirrored by an increase in the current account deficit. In 2007 and 2008, once more there was a change in direction of the movements of trade in goods to the movements in the overall current account balance. In 2009 however, the decrease in the trade in goods deficit once again broadly matched the fall in the current account deficit.

The last trade in goods surplus, recorded in 1982, contributed to a current account surplus. Following 1982, the goods balance went into deficit and this increased to a peak of $f 24.7$ billion in 1989, while the current balance deteriorated to a deficit of $\mathrm{f} 25.5$ billion. From 1989 until the late 1990s, both the trade in goods and current account deficits broadly fell and then subsequently rose. From 2001 to 2003, while the goods deficit continued to grow, the current account deficit narrowed due to a widening income surplus. From 2004 the deficit on trade in goods increased, matched by a rise in the current account deficit. In 2007 and 2008 however, the increasing deficit on trade in goods was more than offset by increasing surpluses on both trade in services and income. This reduced the current account deficit by $\mathrm{f} 8.5$ billion in 2007 and by a further $£ 12.7$ billion in 2008. In 2009 the $£ 11.2$ billion decrease in the deficit for Trade in Goods drove the $f 8.3$ billion decline in the current account deficit.

\section{Trade in goods and services}

The trade in goods account recorded net surpluses in the years 1980 to 1982, largely as a result of growth in exports of North Sea oil. Since then however, the trade in goods account has remained in deficit. The deficit grew significantly in the late 1980 s to reach a peak of $£ 24.7$ billion in 1989 , before narrowing in the 1990s to levels of around $£ 10$ billion to $f 14$ billion. In 1998 the deficit jumped by over $f 9$ billion, and it has

\section{Figure 1.3}

\section{Trade in goods and services Credits less debits \\ $\mathrm{f}$ billion}

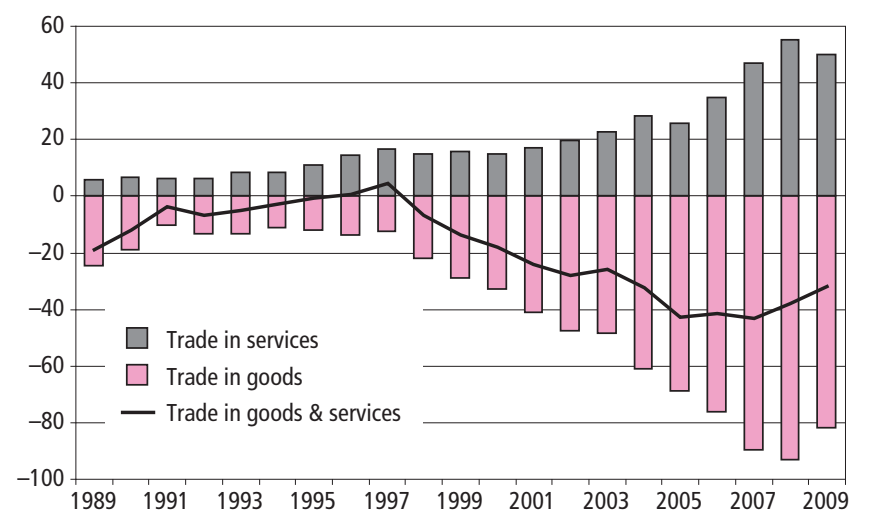


continued to rise reaching a cash record of $£ 93.1$ billion in 2008 before falling to $\mathrm{f} 81.9$ billion in 2009 .

The trade in services account has shown a surplus for every year since 1966. The surplus on services generally increased until 1987, during which time it broadly offset the deficit on trade in goods. From 1988 to 1992 the surplus was reasonably steady at around $\mathrm{f} 6$ billion annually. The services surplus then increased considerably, reaching $\mathrm{f} 16.8$ billion in 1997. It dropped back to $\mathrm{f} 15.0$ billion in 1998 but by 2004 had risen to $\mathrm{f} 28.4$ billion. It fell back slightly in 2005 then rose to a record $£ 55.4$ billion in 2008 before dropping back to $f 49.9$ billion in 2009 .

\section{Income}

The income account consists of compensation of employees and investment income, the latter dominating the account. Historically the balance on compensation of employees has generally been in deficit. It moved into surplus in the late 1990s but moved back into deficit in 2004, where it has remained.

The investment income balance was in surplus until 1976. From 1977 to 1996 it showed a deficit in nearly every year. Then from 1997 onwards, except for 1999, it has shown a surplus. The surplus has mainly been driven by a direct investment income surplus partly offset by deficits on earnings from portfolio investment equity securities, and other investment principally UK banks' net interest payments on deposits. From 2002 to 2004 the surplus was around $\mathrm{f} 18$ billion in each year. In 2005 the surplus rose to $f 22.5$ billion due to net earnings of $£ 43.0$ billion on direct investment, but then dropped sharply in 2006 to $\mathrm{f} 9.4$ billion. Investment income rose in 2007 to $\mathrm{f} 20.8$ billion and to a new record level of $£ 32.0$ billion in 2009. By sector, net earnings of other sectors were $\mathrm{f21.3}$ billion in 2009, compared to $£ 13.5$ billion in 2008 . This increase was partially offset by a $\mathrm{f} 6.3$ billion fall in net earnings by monetary financial institutions (banks and building societies) in 2009.

\section{Figure 1.4}

\section{Investment income \\ Credits less debits}

f billion

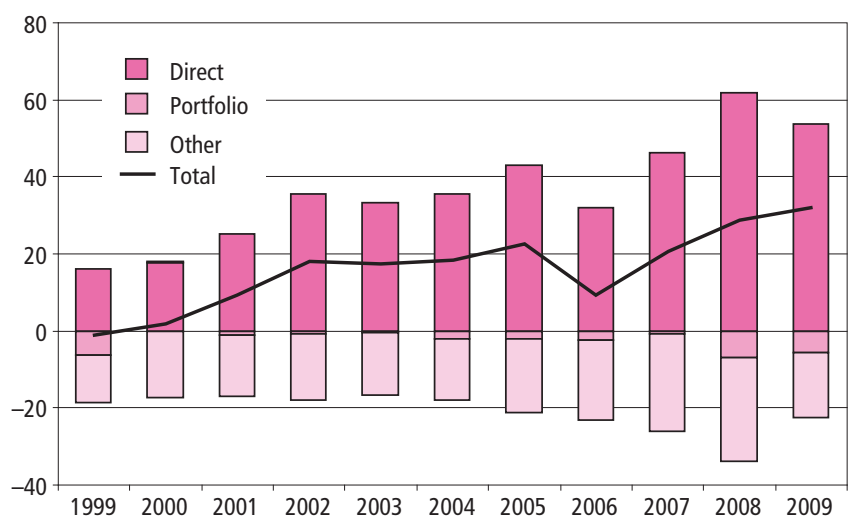

\section{Current transfers}

The transfers account has shown a deficit in every year since 1960. The deficit increased steadily to reach $£ 4.8$ billion in 1990. In 1991 the deficit reduced to $f 1.0$ billion, reflecting $£ 2.1$ billion receipts from other countries towards the UK's cost of the first Gulf conflict. The deficit has since increased, to reach a record $£ 14.8$ billion in 2009. Separate data for general government and other sectors are available from 1986 and show that both have been consistently in deficit since 1992. The volatility in this account is driven by fluctuating net contributions to EU Institutions.

\section{Figure 1.5}

\section{Current transfers Credits less debits}

$\mathrm{f}$ billion

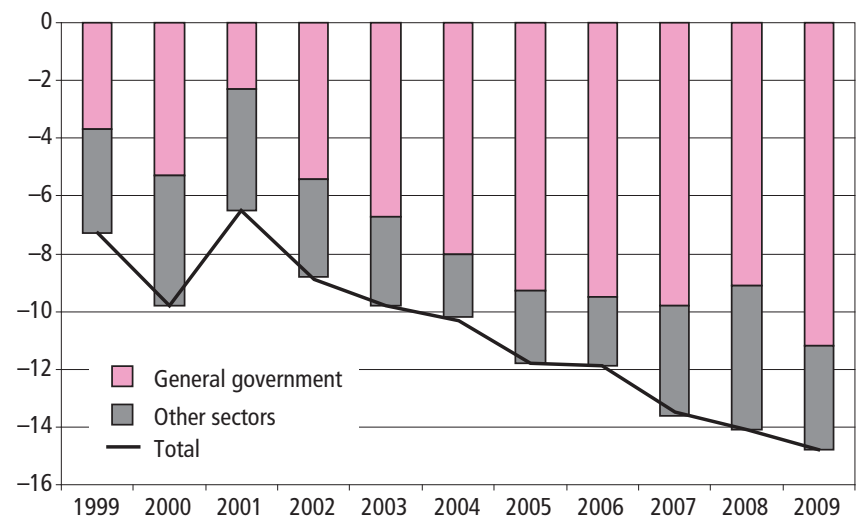

\section{Revisions since Pink Book 2009}

The current account balance has been revised back to 2006 in this publication, as compared with data published in the Pink Book 2009, reflecting the incorporation of GDP balancing adjustments for Trade in Services and annual inquiry results for 2007 and 2008. Details of the sources of these changes are given on pages 17-18 of the Introduction, and the impact of the changes can be seen in Figure 1.6 and in Table 1.1R.

\section{Investment flows, levels and income}

One important set of relationships within the balance of payments is the link between the financial account (investment flows), the international investment position (levels or balance sheets), and the income deriving from the balance sheets. This is explained in more detail in the Introduction. Although a reconciliation statement between opening and closing levels and flows is not officially compiled in the UK, Table 1.3 shows the rudiments of this relationship over the years for which consistent detailed data are available. Within the three main categories of investment (direct, portfolio and other), as well as reserve assets, it can be seen that the difference in the values 


\section{Figure 1.6}

\section{Revisions since Pink Book 2009 \\ Credits less debits \\ f billion}

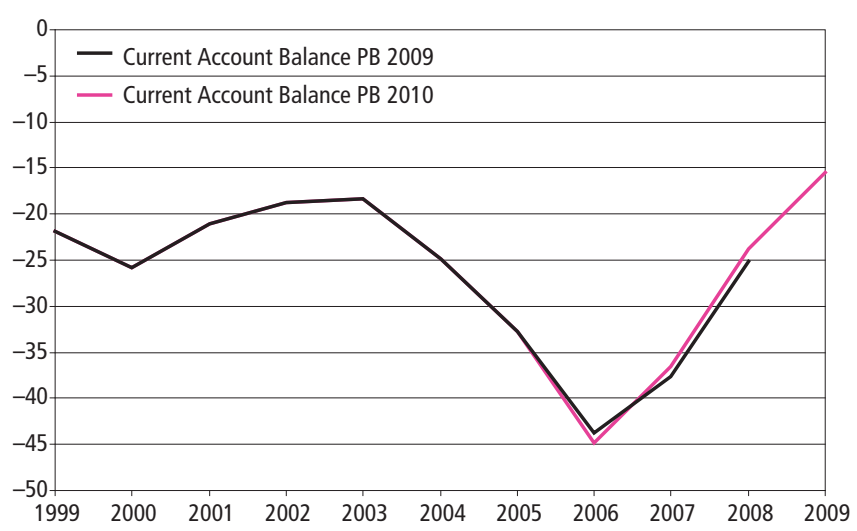

Figure 1.7

\section{International investment position and income} Credits less debits

f billion (IIP)

$\mathrm{f}$ billion (income)

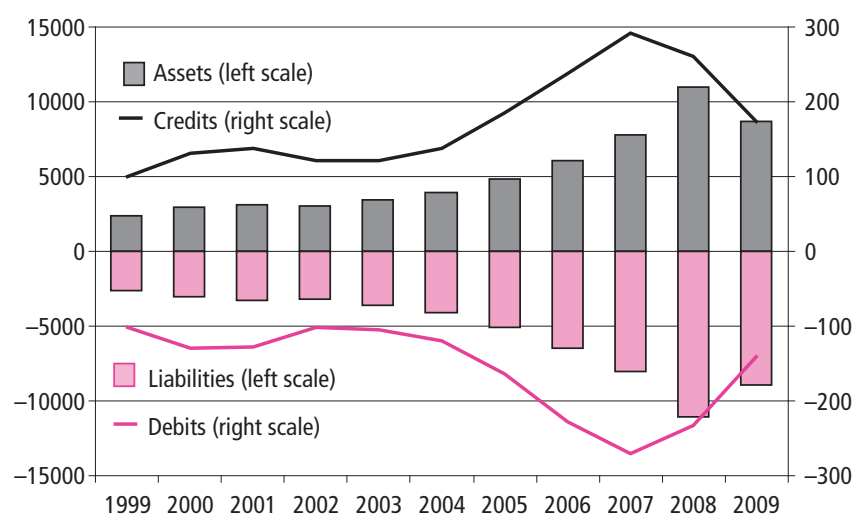

of the balance sheet at the end of one year and the previous year is approximately equal to the value of the financial transactions in that year. The difference between the two amounts is explained by valuation, exchange rate and other effects such as company write-offs.

The value of both external assets and liabilities in the international investment position has been rising steadily since 1980, reflecting both the increased global investment and the increasing prices of external assets and liabilities. Except for 1990, the UK's external assets exceeded external liabilities in every year until 1995. Between 1995 and 2009, external liabilities exceeded external assets.

Since 1995, there has been approaching a sixfold increase in the levels of both external assets and liabilities. At the end of 2009 external assets stood at $£ 8.7$ trillion, while external liabilities stood at $\mathrm{f} 9.0$ trillion, this resulted in a net liability position of $\mathrm{f} 275.0$ billion.

\section{Implied 'rates of return'}

Another important relationship is that which exists between investment income and the international investment position. This can be considered most easily by looking at the implied 'rates of return' for both assets and liabilities. In total, the implied rate of return on liabilities was higher than on assets from the late 1970s until the mid 1990s. Since 1997, although the return on assets has been higher, both have been at relatively low levels.

The rates of return for direct investment are usually significantly higher than for other forms of investment although the rates dropped considerably in 2008: 6.9 per cent for assets and 0.9 per cent for liabilities. The rates of return rebounded somewhat in 2009 but remained relatively low compared with the earlier parts of the decade. Historically, the relatively higher return is probably a consequence, in part, of comparatively lower valuations since direct investment levels are at book value rather than market value used elsewhere, but may also reflect the higher return required to make the longer term investment worthwhile. In recent years however, as the global recession has taken hold, the implied rate of return for direct investment has contracted considerably. Between 2006 and 2009 the implied rate of return for direct investment assets dropped from 11.6 per cent to 7.1 per cent and liabilities declined from 9.6 per cent to 2.9 per cent.

Within portfolio investment, rates of return on debt securities have generally been higher than on equity securities. From 1998 to 2003 the difference in the rate of returns generally narrowed before widening in more recent years, and declining once again in 2008 and 2009. This was due to the rate of return on equity continuing to grow, while the rate of return on debt declined as interest rates fell in 2008, then both declined in 2009.

Rates of return on other investment were similar to returns on debt in the early 1990s, but between then and 2004 they were

\section{Figure 1.8}

\section{Implied rates of return on assets}

Per cent

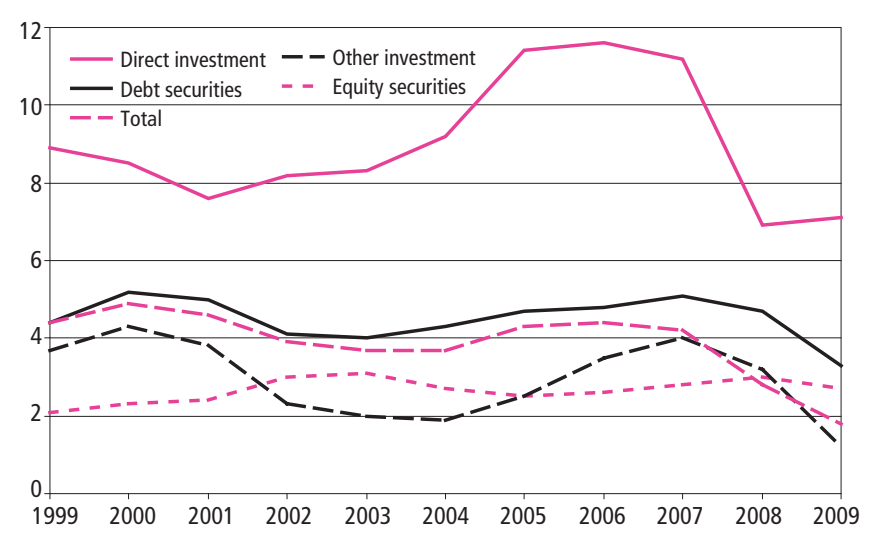


Figure 1.9

Implied rates of return on liabilities

Per cent

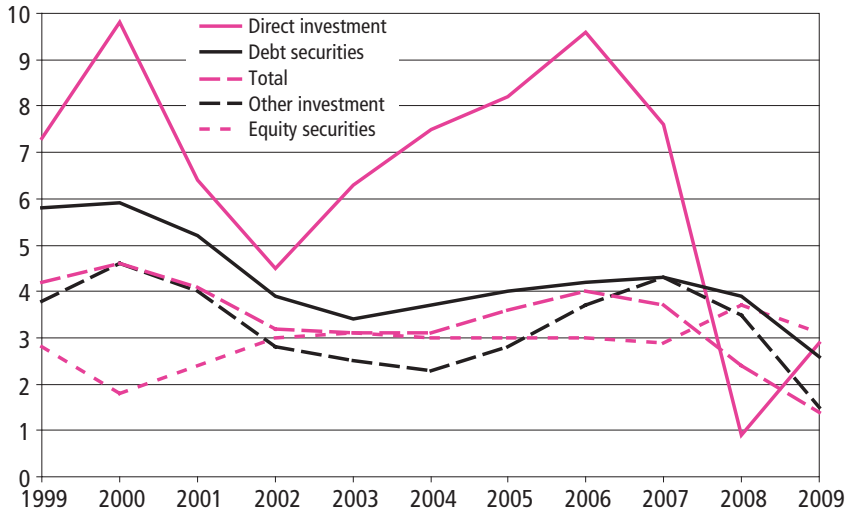

generally in decline. From 2004 until 2007 they began to increase again, but started to declined in 2008 before dropping to a low of 1.2 per cent for assets and 1.5 per cent for liabilities in 2009. Given that other investment constitutes approaching half of the value of the balance sheets, it is not surprising that the rates of return have reflected the movements in interest rates on loans and deposits such as the base rate and the London Interbank Offered Rate (LIBOR). 


\section{Summary of balance of payments \\ - Balances (credits less debits)}

$£$ million

\begin{tabular}{|c|c|c|c|c|c|c|c|c|c|c|c|c|}
\hline & \multicolumn{9}{|c|}{ Current account } & \multirow[b]{2}{*}{$\begin{array}{r}\text { Capital } \\
\text { account }\end{array}$} & \multirow[b]{2}{*}{$\begin{array}{r}\text { Financial } \\
\text { account }\end{array}$} & \multirow[b]{2}{*}{$\begin{array}{r}\text { Net errors } \\
\& \text { omissions }\end{array}$} \\
\hline & $\begin{array}{r}\text { Trade in } \\
\text { goods }\end{array}$ & $\begin{array}{l}\text { Trade in } \\
\text { services }\end{array}$ & $\begin{array}{r}\text { Total goods } \\
\text { and } \\
\text { services }\end{array}$ & $\begin{array}{r}\text { Compensati- } \\
\text { on of } \\
\text { employees }\end{array}$ & $\begin{array}{r}\text { Investment } \\
\text { income }\end{array}$ & $\begin{array}{r}\text { Total } \\
\text { income }\end{array}$ & $\begin{array}{l}\text { Current } \\
\text { transfers }\end{array}$ & $\begin{array}{l}\text { Current } \\
\text { balance }\end{array}$ & $\begin{array}{r}\text { Current } \\
\text { balance as } \\
\% \text { of } G D P^{1}\end{array}$ & & & \\
\hline $\begin{array}{l}1947 \\
1948 \\
1949 \\
1950 \\
1951\end{array}$ & $\begin{array}{r}\text { LQCT } \\
-358 \\
-152 \\
-137 \\
-54 \\
-692\end{array}$ & $\begin{array}{r}\text { KTMS } \\
-197 \\
-64 \\
-43 \\
-4 \\
32\end{array}$ & $\begin{array}{r}\text { KTMY } \\
-555 \\
-216 \\
-180 \\
-58 \\
-660\end{array}$ & $\begin{array}{r}\text { KTMP } \\
-19 \\
-20 \\
-20 \\
-21 \\
-21\end{array}$ & $\begin{array}{r}\text { HMBM } \\
140 \\
223 \\
206 \\
378 \\
322\end{array}$ & $\begin{array}{r}\text { HMBP } \\
121 \\
203 \\
186 \\
357 \\
301\end{array}$ & $\begin{array}{r}\text { KTNF } \\
123 \\
96 \\
29 \\
39 \\
29\end{array}$ & $\begin{array}{r}\text { HBOG } \\
-311 \\
83 \\
35 \\
338 \\
-330\end{array}$ & $\begin{array}{r}\text { AA6H } \\
0.7 \\
0.3 \\
2.6 \\
-2.3\end{array}$ & $\begin{array}{r}\text { FKMJ } \\
-21 \\
-17 \\
-12 \\
-10 \\
-15\end{array}$ & $\begin{array}{r}\text { HBNT } \\
552 \\
-58 \\
-103 \\
-447 \\
426\end{array}$ & $\begin{array}{r}\mathrm{HHDH} \\
-220 \\
-8 \\
80 \\
119 \\
-81\end{array}$ \\
\hline $\begin{array}{l}1952 \\
1953 \\
1954 \\
1955 \\
1956\end{array}$ & $\begin{array}{r}-272 \\
-244 \\
-210 \\
-315 \\
50\end{array}$ & $\begin{array}{r}123 \\
123 \\
115 \\
42 \\
26\end{array}$ & $\begin{array}{r}-149 \\
-121 \\
-95 \\
-273 \\
76\end{array}$ & $\begin{array}{l}-22 \\
-25 \\
-27 \\
-27 \\
-30\end{array}$ & $\begin{array}{l}231 \\
207 \\
227 \\
149 \\
203\end{array}$ & $\begin{array}{l}209 \\
182 \\
200 \\
122 \\
173\end{array}$ & $\begin{array}{r}169 \\
143 \\
55 \\
43 \\
2\end{array}$ & $\begin{array}{r}229 \\
204 \\
160 \\
-108 \\
251\end{array}$ & $\begin{array}{r}1.4 \\
1.2 \\
0.9 \\
-0.6 \\
1.2\end{array}$ & $\begin{array}{l}-15 \\
-13 \\
-13 \\
-15 \\
-13\end{array}$ & $\begin{array}{r}-229 \\
-177 \\
-174 \\
34 \\
-250\end{array}$ & $\begin{array}{r}15 \\
-14 \\
27 \\
89 \\
12\end{array}$ \\
\hline $\begin{array}{l}1957 \\
1958 \\
1959 \\
1960 \\
1961\end{array}$ & $\begin{array}{r}-29 \\
34 \\
-116 \\
-404 \\
-144\end{array}$ & $\begin{array}{r}121 \\
119 \\
118 \\
39 \\
51\end{array}$ & $\begin{array}{r}92 \\
153 \\
2 \\
-365 \\
-93\end{array}$ & $\begin{array}{l}-32 \\
-34 \\
-37 \\
-35 \\
-35\end{array}$ & $\begin{array}{l}223 \\
261 \\
233 \\
201 \\
223\end{array}$ & $\begin{array}{l}191 \\
227 \\
196 \\
166 \\
188\end{array}$ & $\begin{array}{r}-5 \\
4 \\
- \\
-6 \\
-9\end{array}$ & $\begin{array}{r}278 \\
384 \\
198 \\
-205 \\
86\end{array}$ & $\begin{array}{r}1.3 \\
1.7 \\
0.8 \\
-0.8 \\
0.3\end{array}$ & $\begin{array}{r}-13 \\
-10 \\
-5 \\
-6 \\
-12\end{array}$ & $\begin{array}{r}-313 \\
-411 \\
-68 \\
-7 \\
23\end{array}$ & $\begin{array}{r}48 \\
37 \\
-125 \\
218 \\
-97\end{array}$ \\
\hline $\begin{array}{l}1962 \\
1963 \\
1964 \\
1965 \\
1966\end{array}$ & $\begin{array}{l}-104 \\
-123 \\
-551 \\
-263 \\
-111\end{array}$ & $\begin{array}{r}50 \\
4 \\
-34 \\
-66 \\
44\end{array}$ & $\begin{array}{r}-54 \\
-119 \\
-585 \\
-329 \\
-67\end{array}$ & $\begin{array}{l}-37 \\
-38 \\
-33 \\
-34 \\
-39\end{array}$ & $\begin{array}{l}301 \\
364 \\
365 \\
405 \\
358\end{array}$ & $\begin{array}{l}264 \\
326 \\
332 \\
371 \\
319\end{array}$ & $\begin{array}{l}-14 \\
-37 \\
-74 \\
-75 \\
-91\end{array}$ & $\begin{array}{r}196 \\
170 \\
-327 \\
-33 \\
161\end{array}$ & $\begin{array}{r}0.7 \\
0.6 \\
-1.0 \\
-0.1 \\
0.4\end{array}$ & $\begin{array}{l}-12 \\
-16 \\
-17 \\
-18 \\
-19\end{array}$ & $\begin{array}{r}-195 \\
-30 \\
392 \\
49 \\
22\end{array}$ & $\begin{array}{r}11 \\
-124 \\
-48 \\
2 \\
-164\end{array}$ \\
\hline $\begin{array}{l}1967 \\
1968 \\
1969 \\
1970 \\
1971\end{array}$ & $\begin{array}{r}-601 \\
-708 \\
-214 \\
-18 \\
205\end{array}$ & $\begin{array}{l}157 \\
341 \\
392 \\
455 \\
617\end{array}$ & $\begin{array}{r}-444 \\
-367 \\
178 \\
437 \\
822\end{array}$ & $\begin{array}{l}-39 \\
-48 \\
-47 \\
-56 \\
-63\end{array}$ & $\begin{array}{l}354 \\
303 \\
468 \\
527 \\
454\end{array}$ & $\begin{array}{l}315 \\
255 \\
421 \\
471 \\
391\end{array}$ & $\begin{array}{r}-118 \\
-119 \\
-109 \\
-89 \\
-90\end{array}$ & $\begin{array}{r}-247 \\
-231 \\
490 \\
819 \\
1123\end{array}$ & $\begin{array}{r}-0.6 \\
-0.5 \\
1.0 \\
1.6 \\
2.0\end{array}$ & $\begin{array}{l}-25 \\
-26 \\
-23 \\
-22 \\
-23\end{array}$ & $\begin{array}{r}179 \\
688 \\
-794 \\
-818 \\
-1330\end{array}$ & $\begin{array}{r}93 \\
-431 \\
327 \\
21 \\
230\end{array}$ \\
\hline $\begin{array}{l}1972 \\
1973 \\
1974 \\
1975 \\
1976\end{array}$ & $\begin{array}{r}-736 \\
-2573 \\
-5241 \\
-3245 \\
-3930\end{array}$ & $\begin{array}{r}722 \\
907 \\
1292 \\
1708 \\
2872\end{array}$ & $\begin{array}{l}-14 \\
-1666 \\
-3949 \\
-1537 \\
-1058\end{array}$ & $\begin{array}{r}-52 \\
-68 \\
-92 \\
-102 \\
-140\end{array}$ & $\begin{array}{r}350 \\
970 \\
1010 \\
257 \\
760\end{array}$ & $\begin{array}{l}298 \\
902 \\
918 \\
155 \\
620\end{array}$ & $\begin{array}{l}-142 \\
-336 \\
-302 \\
-313 \\
-534\end{array}$ & $\begin{array}{r}142 \\
-1100 \\
-3333 \\
-1695 \\
-972\end{array}$ & $\begin{array}{r}0.2 \\
-1.5 \\
-4.0 \\
-1.6 \\
-0.8\end{array}$ & $\begin{array}{l}-35 \\
-39 \\
-34 \\
-36 \\
-12\end{array}$ & $\begin{array}{r}477 \\
1031 \\
3185 \\
1569 \\
507\end{array}$ & $\begin{array}{r}-584 \\
108 \\
182 \\
162 \\
477\end{array}$ \\
\hline $\begin{array}{l}1977 \\
1978 \\
1979 \\
1980 \\
1981\end{array}$ & $\begin{array}{rl}-2 & 271 \\
-1 & 534 \\
-3326 \\
1329 \\
3238\end{array}$ & $\begin{array}{l}3704 \\
4215 \\
4573 \\
4414 \\
4776\end{array}$ & $\begin{array}{l}1433 \\
2681 \\
1247 \\
5743 \\
8014\end{array}$ & $\begin{array}{r}-152 \\
-140 \\
-130 \\
-82 \\
-66\end{array}$ & $\begin{array}{r}-678 \\
-300 \\
-342 \\
-2268 \\
-1883\end{array}$ & $\begin{array}{r}-830 \\
-440 \\
-472 \\
-2350 \\
-1949\end{array}$ & $\begin{array}{r}-889 \\
-1420 \\
-1777 \\
-1653 \\
-1219\end{array}$ & $\begin{array}{r}-286 \\
821 \\
-1002 \\
1740 \\
4846\end{array}$ & $\begin{array}{r}-0.2 \\
0.5 \\
-0.5 \\
0.8 \\
1.9\end{array}$ & $\begin{array}{r}11 \\
-79 \\
-103 \\
-4 \\
-79\end{array}$ & $\begin{array}{r}-3286 \\
-2655 \\
864 \\
-2157 \\
-5312\end{array}$ & $\begin{array}{r}3561 \\
1913 \\
241 \\
421 \\
545\end{array}$ \\
\hline $\begin{array}{l}1987 \\
1988 \\
1989 \\
1990 \\
1991\end{array}$ & $\begin{array}{l}-11698 \\
-21553 \\
-24724 \\
-18707 \\
-10223\end{array}$ & $\begin{array}{l}8604 \\
6388 \\
5 \quad 866 \\
6643 \\
6312\end{array}$ & $\begin{array}{r}-3094 \\
-15165 \\
-18858 \\
-12064 \\
-3911\end{array}$ & $\begin{array}{r}-174 \\
-64 \\
-138 \\
-110 \\
-63\end{array}$ & $\begin{array}{r}-730 \\
-1188 \\
-2309 \\
-4586 \\
-5642\end{array}$ & $\begin{array}{r}-904 \\
-1252 \\
-2447 \\
-4696 \\
-5705\end{array}$ & $\begin{array}{r}-3437 \\
-3293 \\
-4228 \\
-4802 \\
-999\end{array}$ & $\begin{array}{r}-7435 \\
-19710 \\
-25533 \\
-21562 \\
-10615\end{array}$ & $\begin{array}{l}-1.7 \\
-4.1 \\
-4.9 \\
-3.8 \\
-1.8\end{array}$ & $\begin{array}{l}333 \\
235 \\
270 \\
497 \\
290\end{array}$ & $\begin{array}{r}10606 \\
16989 \\
13614 \\
22272 \\
7855\end{array}$ & $\begin{array}{r}-3504 \\
2486 \\
11649 \\
-1207 \\
2470\end{array}$ \\
\hline $\begin{array}{l}1992 \\
1993 \\
1994 \\
1995 \\
1996\end{array}$ & $\begin{array}{l}-13050 \\
-13066 \\
-11126 \\
-12023 \\
-13722\end{array}$ & $\begin{array}{r}6353 \\
8174 \\
8161 \\
11165 \\
14312\end{array}$ & $\begin{array}{r}-6697 \\
-4892 \\
-2965 \\
-858 \\
590\end{array}$ & $\begin{array}{r}-49 \\
35 \\
-170 \\
-296 \\
93\end{array}$ & $\begin{array}{r}-1037 \\
-2547 \\
1521 \\
-546 \\
-2460\end{array}$ & $\begin{array}{r}-1086 \\
-2512 \\
1351 \\
-842 \\
-2367\end{array}$ & $\begin{array}{l}-5228 \\
-5056 \\
-5187 \\
-7363 \\
-4539\end{array}$ & $\begin{array}{r}-13011 \\
-12460 \\
-6801 \\
-9063 \\
-6316\end{array}$ & $\begin{array}{l}-2.1 \\
-1.9 \\
-1.0 \\
-1.2 \\
-0.8\end{array}$ & $\begin{array}{r}421 \\
309 \\
33 \\
533 \\
1260\end{array}$ & $\begin{array}{r}16311 \\
22278 \\
-3240 \\
-1717 \\
-940\end{array}$ & $\begin{array}{r}-3721 \\
-10127 \\
10008 \\
10247 \\
5996\end{array}$ \\
\hline $\begin{array}{l}1997 \\
1998 \\
1999 \\
2000 \\
2001\end{array}$ & $\begin{array}{l}-12342 \\
-21813 \\
-29051 \\
-32976 \\
-41212\end{array}$ & $\begin{array}{l}16801 \\
15003 \\
15562 \\
15002 \\
17200\end{array}$ & $\begin{array}{r}4459 \\
-6810 \\
-13489 \\
-17974 \\
-24012\end{array}$ & $\begin{array}{r}83 \\
-10 \\
201 \\
150 \\
66\end{array}$ & $\begin{array}{r}241 \\
11813 \\
-1244 \\
1812 \\
9359\end{array}$ & $\begin{array}{r}324 \\
11803 \\
-1043 \\
1962 \\
9425\end{array}$ & $\begin{array}{l}-5745 \\
-8172 \\
-7322 \\
-9775 \\
-6515\end{array}$ & $\begin{array}{r}-962 \\
-3179 \\
-21854 \\
-25787 \\
-21102\end{array}$ & $\begin{array}{l}-0.1 \\
-0.4 \\
-2.4 \\
-2.6 \\
-2.1\end{array}$ & $\begin{array}{r}958 \\
489 \\
747 \\
1703 \\
1318\end{array}$ & $\begin{array}{r}-7294 \\
4480 \\
29505 \\
23133 \\
27194\end{array}$ & $\begin{array}{r}7298 \\
-1790 \\
-8398 \\
951 \\
-7410\end{array}$ \\
\hline $\begin{array}{l}2002 \\
2003 \\
2004 \\
2005 \\
2006\end{array}$ & $\begin{array}{l}-47705 \\
-48607 \\
-60900 \\
-68589 \\
-76312\end{array}$ & $\begin{array}{l}19632 \\
22612 \\
28414 \\
25742 \\
34775\end{array}$ & $\begin{array}{l}-28073 \\
-25995 \\
-32486 \\
-42847 \\
-41537\end{array}$ & $\begin{array}{r}67 \\
59 \\
-494 \\
-610 \\
-958\end{array}$ & $\begin{array}{r}18219 \\
17464 \\
18339 \\
22465 \\
9439\end{array}$ & $\begin{array}{r}18286 \\
17523 \\
17845 \\
21855 \\
8481\end{array}$ & $\begin{array}{r}-8870 \\
-9835 \\
-10276 \\
-11849 \\
-11878\end{array}$ & $\begin{array}{l}-18657 \\
-18307 \\
-24917 \\
-32841 \\
-44934\end{array}$ & $\begin{array}{l}-1.7 \\
-1.6 \\
-2.1 \\
-2.6 \\
-3.4\end{array}$ & $\begin{array}{r}932 \\
1466 \\
2064 \\
1503 \\
975\end{array}$ & $\begin{array}{l}24204 \\
22553 \\
29358 \\
29024 \\
41974\end{array}$ & $\begin{array}{r}-6479 \\
-5712 \\
-6505 \\
2314 \\
1985\end{array}$ \\
\hline $\begin{array}{l}2007 \\
2008 \\
2009\end{array}$ & $\begin{array}{l}-89754 \\
-93116 \\
-81875\end{array}$ & $\begin{array}{l}46798 \\
55356 \\
49852\end{array}$ & $\begin{array}{l}-42956 \\
-37760 \\
-32023\end{array}$ & $\begin{array}{l}-734 \\
-715 \\
-688\end{array}$ & $\begin{array}{l}20754 \\
28750 \\
31970\end{array}$ & $\begin{array}{l}20020 \\
28035 \\
31282\end{array}$ & $\begin{array}{l}-13546 \\
-14051 \\
-14765\end{array}$ & $\begin{array}{l}-36482 \\
-23776 \\
-15506\end{array}$ & $\begin{array}{l}-2.6 \\
-1.6 \\
-1.1\end{array}$ & $\begin{array}{l}2566 \\
3241 \\
3219\end{array}$ & $\begin{array}{r}24790 \\
25811 \\
5597\end{array}$ & $\begin{array}{r}9126 \\
-5276 \\
6690\end{array}$ \\
\hline
\end{tabular}

1 Using series YBHA: GDP at current market prices. 


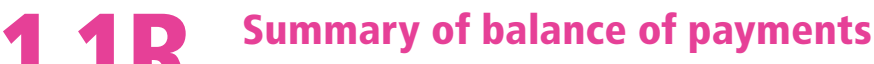 \\ Revisions since ONS Pink Book 2009}

$£$ million

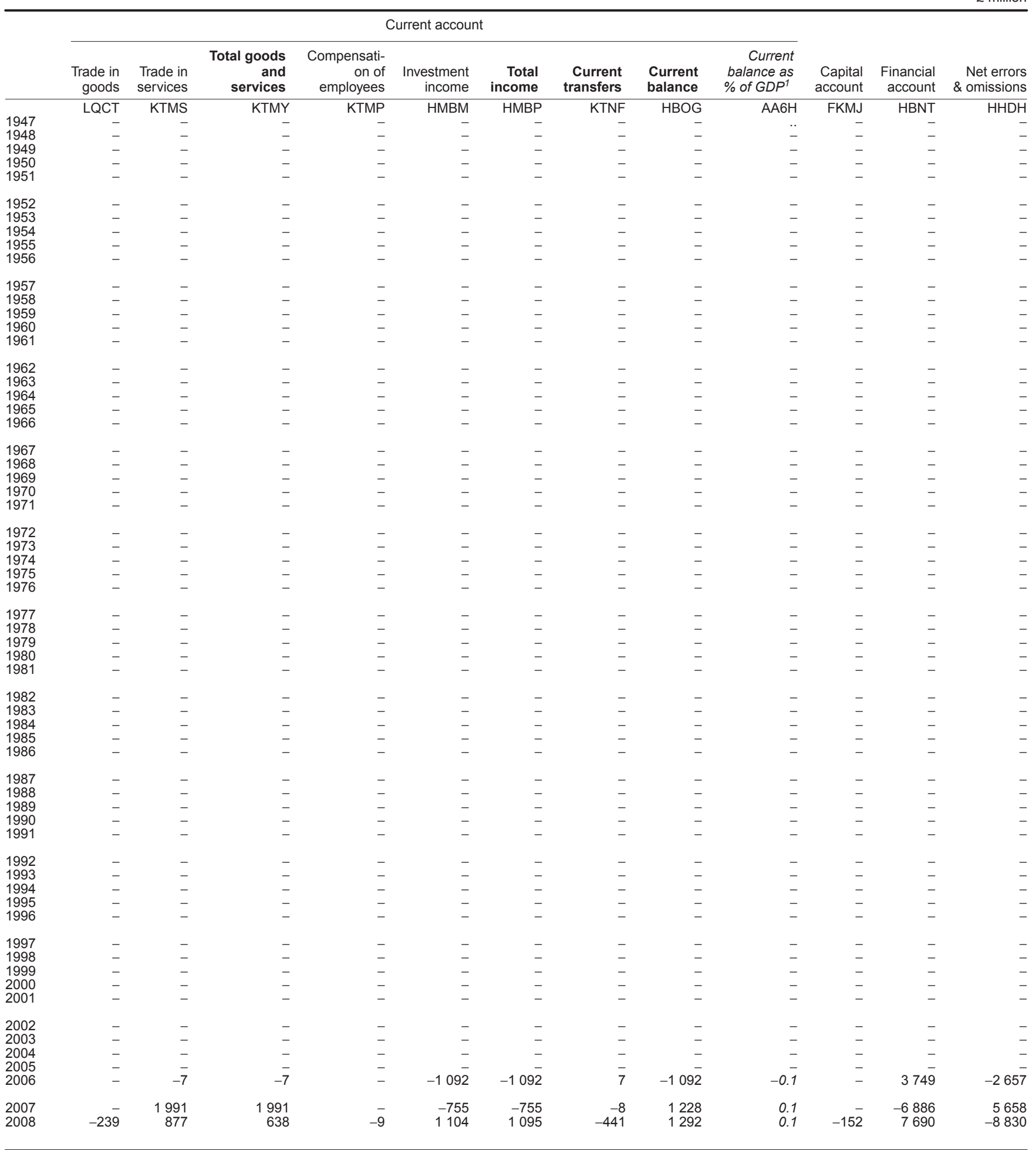

1 Using series YBHA: GDP at current market prices. 


\begin{tabular}{|c|c|c|c|c|c|c|c|c|c|c|c|c|}
\hline & & 1988 & 1989 & 1990 & 1991 & 1992 & 1993 & 1994 & 1995 & 1996 & 1997 & 1998 \\
\hline \multicolumn{13}{|l|}{ Credits } \\
\hline \multicolumn{13}{|l|}{ Exports of goods and services } \\
\hline Exports of goods & LQAD & 80711 & 92611 & 102313 & 103939 & 107863 & 122229 & 135143 & 153577 & 167196 & 171923 & 164056 \\
\hline Total exports of goods and services & KTMW & 109804 & 124153 & 136583 & 138662 & 145480 & 165834 & 183215 & 207147 & 229047 & 237478 & 233284 \\
\hline \multicolumn{13}{|l|}{ Income } \\
\hline Investment income & HMBN & 54540 & 71536 & 76353 & 73652 & 65168 & 70944 & 72585 & 85490 & 89794 & 93360 & 102551 \\
\hline Total income & HMBQ & 54985 & 72012 & 76896 & 74203 & 65719 & 71539 & 73266 & 86377 & 90705 & 94367 & 103391 \\
\hline \multicolumn{13}{|l|}{ Current transfers } \\
\hline General government & FJUM & 1929 & 1507 & 2050 & 4892 & 2180 & 2826 & 2138 & 1730 & 2828 & 2173 & 1767 \\
\hline Other sectors & FUUN & 4584 & 5244 & 6025 & 7611 & 10397 & 9612 & 9521 & 10891 & 13371 & 10735 & 10682 \\
\hline Total current transfers & KTND & 6513 & 6751 & 8075 & 12503 & 12577 & 12438 & 11659 & 12621 & 16199 & 12908 & 12449 \\
\hline Total & HBOE & 171302 & 202916 & 221554 & 225368 & 223776 & 249811 & 268140 & 306145 & 335951 & 344753 & 349124 \\
\hline \multicolumn{13}{|l|}{ Debits } \\
\hline \multicolumn{13}{|l|}{ Imports of goods and services } \\
\hline Imports of goods & LQBL & 102264 & 117335 & 121020 & 114162 & 120913 & 135295 & 146269 & 165600 & 180918 & 184265 & 185869 \\
\hline Imports of services & KTMR & 22705 & 25676 & 27627 & 28411 & 31264 & 35431 & 39911 & 42405 & 47539 & 48754 & 54225 \\
\hline Total imports of goods and services & KTMX & 124969 & 143011 & 148647 & 142573 & 152177 & 170726 & 186180 & 208005 & 228457 & 233019 & 240094 \\
\hline \multicolumn{13}{|l|}{ Income } \\
\hline Compensation of employees & Ктмо & 509 & 614 & 653 & 614 & 600 & 560 & 851 & 1183 & 818 & 924 & 850 \\
\hline Investment income & HMBO & 55728 & 73845 & 80939 & 79294 & 66205 & 73491 & 71064 & 86036 & 92254 & 93119 & 90738 \\
\hline Total income & HMBR & 56237 & 74459 & 81592 & 79908 & 66805 & 74051 & 71915 & 87219 & 93072 & 94043 & 91588 \\
\hline \multicolumn{13}{|l|}{ Current transfers } \\
\hline General government & FUUO & 2226 & 2055 & 1995 & 3218 & 3506 & 4156 & 4795 & 4811 & 5081 & 5087 & 6585 \\
\hline Total & HBOF & 191012 & 228449 & 243116 & 235983 & 236787 & 262271 & 274941 & 315208 & 342267 & 345715 & 352303 \\
\hline \multicolumn{13}{|l|}{ Balances } \\
\hline \multicolumn{13}{|l|}{ Trade in goods and services } \\
\hline Trade in goods & LQCT & -21553 & -24724 & -18707 & -10223 & -13050 & -13066 & -11126 & -12023 & -13722 & -12342 & -21813 \\
\hline Trade in services & KTMS & 6388 & 5866 & 6643 & 6312 & 6353 & 8174 & 8161 & 11165 & 14312 & 16801 & 15003 \\
\hline Total trade in goods and services & KTMY & -15165 & -18858 & -12064 & -3911 & -6697 & -4892 & -2965 & -858 & 590 & 4459 & -6810 \\
\hline \multicolumn{13}{|l|}{ Income } \\
\hline Compensation of employees & KTMP & -64 & -138 & -110 & -63 & -49 & & -170 & -296 & & 83 & -10 \\
\hline Investment income & HMBM & -1188 & -2309 & -4586 & -5642 & -1037 & -2547 & 1521 & -546 & -2460 & 241 & 11813 \\
\hline Total income & HMBP & -1252 & -2447 & -4696 & -5705 & -1086 & -2512 & 1351 & -842 & -2367 & 324 & 11803 \\
\hline \multicolumn{13}{|l|}{ Current transfers } \\
\hline General government & FUUQ & -297 & -548 & 55 & 1674 & -1326 & -1330 & -2657 & -3081 & -2253 & -2914 & -4818 \\
\hline Other sectors & FUUR & -2996 & -3680 & -4857 & -2673 & -3902 & -3726 & -2530 & -4282 & -2286 & -2831 & -3354 \\
\hline Total current transfers & KTNF & -3293 & -4228 & -4802 & -999 & -5228 & -5056 & -5187 & -7363 & -4539 & -5745 & -8172 \\
\hline Total (Current balance) & HBOG & -19710 & -25533 & -21562 & -10615 & -13011 & -12460 & -6801 & -9063 & -6316 & -962 & -3179 \\
\hline
\end{tabular}




$\begin{array}{lllllllllll}1999 & 2000 & 2001 & 2002 & 2003 & 2004 & 2005 & 2006 & 2007 & 2008 & 2009\end{array}$

Credits

Exports of goods and services

Exports of goods

Exports of services

LQAD KTMQ

Total exports of goods and services KTMW

Income

Compensation of employees Investment income

Total income

KTMN HMBN

HMBQ

Current transfers

General government

Other sectors

Total current transfers

Total

Debits

Imports of goods and services

Imports of services

XTR

Total imports of goods and services KTMX

Income

Compensation of employees

Investment income

Total income

KTMO

HMBO

HMBR

Current transfers

General government

Other sectors

Total current transfers

Total

Balances

Trade in goods and services

Trade in

Trade in services

Total trade in goods and services

IOCT

KTMS

KTMY

Income

Compensation of employees

Investment income

Total income

KTMP

HMBM

HMBP

\begin{tabular}{rrrrrrrrrrr}
201 & 150 & 66 & 67 & 59 & -494 & -610 & -958 & -734 & -715 & -688 \\
-1244 & 1812 & 9359 & 18219 & 17464 & 18339 & 22465 & 9439 & 20754 & 28750 & 31970 \\
\hline-1043 & 1962 & 9425 & 18286 & 17523 & 17845 & 21855 & 8481 & 20020 & 28035 & 31282
\end{tabular}

Current transfers

General government

Other sectors

Total current transfers

FUUQ

FUUR

KTNF

HBOG

$\begin{array}{lllllllllllll}166166 & 187936 & 189093 & 186524 & 188320 & 190874 & 211608 & 243633 & 220858 & 252086 & 227537\end{array}$ $\begin{array}{lllllllllllll}76 & 525 & 81883 & 87773 & 94012 & 102357 & 112922 & 119186 & 134393 & 153145 & 170819 & 159 & 111\end{array}$

$\begin{array}{lllllllllll}242691 & 269819 & 276866 & 280536 & 290677 & 303796 & 330794 & 378026 & 374003 & 422905 & 386 \\ 648\end{array}$

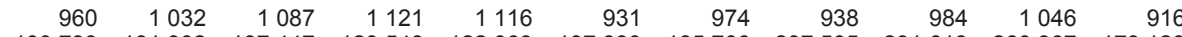
$\begin{array}{llllllllllll}131902 & 137447 & 120543 & 122069 & 137380 & 185766 & 237505 & 291618 & 260967 & 173 & 128\end{array}$

$\begin{array}{lllllllllll}101693 & 132934 & 138534 & 121664 & 123185 & 138311 & 186740 & 238443 & 292602 & 262013 & 174044\end{array}$

\begin{tabular}{rrrrrrrrrrr}
3542 & 2465 & 4991 & 3663 & 3968 & 4177 & 4294 & 4383 & 4318 & 5652 & 6158 \\
8510 & 8018 & 8926 & 8571 & 8079 & 9590 & 13106 & 13789 & 9559 & 10669 & 10465 \\
\hline 12052 & 10483 & 13917 & 12234 & 12047 & 13767 & 17400 & 18172 & 13877 & 16321 & 16623
\end{tabular}

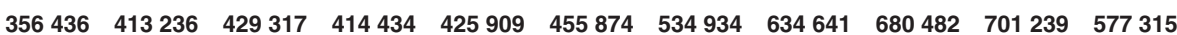

$\begin{array}{llllllllllll}195217 & 220912 & 230305 & 234229 & 236927 & 251774 & 280197 & 319945 & 310612 & 345202 & 309412\end{array}$ $\begin{array}{lllllllllll}60963 & 66881 & 70573 & 74380 & 79745 & 84508 & 93444 & 99618 & 106347 & 115463 & 109259\end{array}$

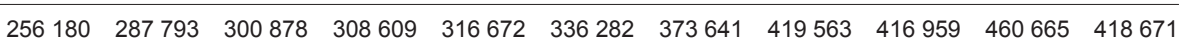

$\begin{array}{lllllllllll}759 & 882 & 1021 & 1054 & 1057 & 1425 & 1584 & 1896 & 1718 & 1761 & 1604\end{array}$

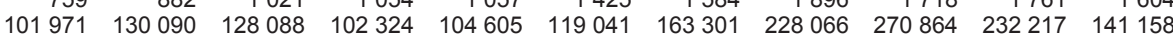

$102736 \quad 130972 \quad 129109 \quad 103378 \quad 105662 \quad 120466 \quad 164885 \quad 229962 \quad 272582 \quad 233978 \quad 142762$

$\begin{array}{lllllllllll}7271 & 7778 & 7340 & 9085 & 10657 & 12225 & 13637 & 13874 & 14082 & 14726 & 17344\end{array}$

\begin{tabular}{llllllllllll}
12 & 103 & 12480 & 13092 & 12019 & 11225 & 11818 & 15612 & 16176 & 13341 & 15646 & 14044 \\
\hline
\end{tabular}

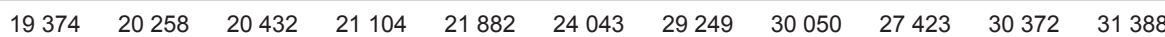

$\begin{array}{lllllllllll}378290 & 439023 & 450419 & 433091 & 444216 & 480791 & 567775 & 679575 & 716964 & 725015 & 592821\end{array}$

\begin{tabular}{rrrrrrrrrrrr}
-29051 & -32976 & -41212 & -47705 & -48607 & -60900 & -68589 & -76312 & -89754 & -93116 & -81875 \\
15562 & 15002 & 17200 & 19632 & 22612 & 28414 & 25742 & 34775 & 46798 & 55356 & 49852 \\
\hline-13489 & -17974 & -24012 & -28073 & -25995 & -32486 & -42847 & -41537 & -42956 & -37760 & -32023
\end{tabular}

Total (Current balance)

\begin{tabular}{rrrrrrrrrrr}
-3729 & -5313 & -2349 & -5422 & -6689 & -8048 & -9343 & -9491 & -9764 & -9074 & -11186 \\
-3593 & -4462 & -4166 & -3448 & -3146 & -2228 & -2506 & -2387 & -3782 & -4977 & -3579 \\
\hline-7322 & -9775 & -6515 & -8870 & -9835 & -10276 & -11849 & -11878 & -13546 & -14051 & -14765
\end{tabular}

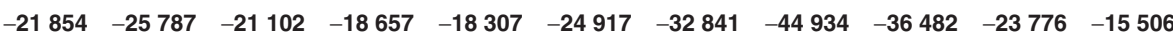




\section{Summary of international investment position, financial account - 3 and investment income}

\begin{tabular}{|c|c|c|c|c|c|c|c|c|c|c|c|c|}
\hline & & 1999 & 2000 & 2001 & 2002 & 2003 & 2004 & 2005 & 2006 & 2007 & 2008 & 2009 \\
\hline \multicolumn{13}{|l|}{ Investment abroad } \\
\hline \multicolumn{13}{|c|}{ International investment position } \\
\hline Direct investment & HBWD & 438.3 & 618.8 & 616.9 & 637.2 & 691.1 & 678.1 & 705.9 & 733.1 & 899.8 & 1046.1 & 1024.4 \\
\hline Portfolio investment & HHZZ & 838.3 & 906.1 & 937.4 & 844.0 & 935.8 & 1092.1 & 1360.9 & 1531.1 & 1697.3 & 1664.3 & 1889.2 \\
\hline Financial derivatives & JX96 & & & & & & & & 853.7 & 1378.1 & 4040.2 & 2201.5 \\
\hline Other investment & HLXV & 1097.3 & 1379.7 & 1521.9 & 1545.2 & 1813.7 & 2118.0 & 2714.8 & 2916.6 & 3744.5 & 4193.6 & 3544.7 \\
\hline Reserve assets & LTEB & 22.2 & 28.8 & 25.6 & 25.5 & 23.8 & 23.2 & 24.7 & 22.9 & 26.7 & 36.3 & 40.1 \\
\hline Total & HBQA & 2396.1 & 2933.4 & 3101.9 & 3051.9 & 3464.5 & 3911.4 & 4806.3 & 6057.4 & 7746.4 & 10980.5 & 8700.0 \\
\hline \multicolumn{13}{|l|}{ Financial account transactions } \\
\hline Direct investment & -HUYP & 125.6 & 155.6 & 42.8 & 35.0 & 40.9 & 51.5 & 44.0 & 45.0 & 162.6 & 87.6 & 30.1 \\
\hline Portfolio investment & $-\mathrm{HHZC}$ & 21.4 & 65.6 & 86.6 & 1.0 & 36.3 & 141.0 & 151.0 & 138.8 & 92.0 & -123.5 & 154.1 \\
\hline Financial derivatives (net) & -ZPNN & -2.7 & -1.6 & -8.4 & -1.0 & 5.4 & 7.9 & -9.6 & -20.6 & 27.0 & 121.7 & -29.1 \\
\hline Other investment & $-X B M M$ & 41.5 & 241.7 & 170.7 & 70.4 & 260.4 & 325.2 & 501.3 & 395.9 & 742.4 & -599.1 & -331.0 \\
\hline Reserve assets & -LTCV & -0.6 & 3.9 & -3.1 & -0.5 & -1.6 & 0.2 & 0.7 & -0.4 & 1.2 & -1.3 & 5.8 \\
\hline Total & - HBNR & 185.2 & 465.2 & 288.5 & 105.0 & 341.4 & 525.8 & 687.3 & 558.7 & 1025.2 & -514.7 & -170.1 \\
\hline Direct investment & HJYW & 33.1 & 45.0 & 46.7 & 51.5 & 55.1 & 63.3 & 79.2 & 83.6 & 91.4 & 67.3 & 73.1 \\
\hline Portfolio investment & HLYX & 25.9 & 33.0 & 34.9 & 32.5 & 32.5 & 36.7 & 45.4 & 55.1 & 66.1 & 67.6 & 54.5 \\
\hline Other investment & AIOP & 40.6 & 52.9 & 54.9 & 35.8 & 33.6 & 36.7 & 60.5 & 98.1 & 133.5 & 125.3 & 44.7 \\
\hline Reserve assets & HHCB & 1.2 & 1.0 & 1.0 & 0.8 & 0.8 & 0.7 & 0.7 & 0.6 & 0.6 & 0.8 & 0.8 \\
\hline Total & HMBN & 100.7 & 131.9 & 137.4 & 120.5 & 122.1 & 137.4 & 185.8 & 237.5 & 291.6 & 261.0 & 173.1 \\
\hline
\end{tabular}

Investment in the UK

International investment position

Portfolio investment

Financial derivatives

Other investment

Total

HBWI

HLXW

JX97

HLYD

HBQB

Financial account transactions

Direct investment

Portfolio investment

Other investment

Total

Investment income

Direct investment

Portfolio investment

Other investment

Total

Net investment

International investment position Direct investment

Portfolio investment

Financial derivatives

Other investment

Reserve assets

Net investment position

Financial account transactions

Direct investment

Portfolio investment

Financial derivatives

Other investment

Reserve assets

Net transactions

Investment income

Direct investment

Portfolio investment

Other investment

Reserve assets

Net earnings

HJYU

HHZF

HBNS

HJYX

HLZC

HLZN

HMBO

HBWQ

CGNH

JX9 8

CGNG

LTEB

HBQC

HJYV

HHZD

ZPNN

HHYR

LTCV

HBNT

HJYE

HLZX

CGNA

HHCB

HMBM

\begin{tabular}{rrrrrrrrrrr}
250.2 & 310.4 & 363.5 & 340.6 & 355.5 & 383.3 & 494.2 & 577.3 & 613.8 & 668.5 & 671.8 \\
933.2 & 1067.6 & 1013.2 & 925.3 & 1082.9 & 1227.9 & 1461.7 & 1703.5 & 1946.0 & 1978.2 & 2376.9 \\
.. &. .0 &.. &.. &.. &.. &.. & 890.5 & 1392.2 & 3915.3 & 2121.9 \\
1400.9 & 1651.6 & 1861.9 & 1906.0 & 2143.2 & 2520.8 & 3103.0 & 3272.0 & 4117.6 & 4520.1 & 3804.4 \\
\hline $\mathbf{2 5 8 4 . 3}$ & $\mathbf{3} \mathbf{0 2 9 . 5}$ & $\mathbf{3} \mathbf{2 3 8 . 5}$ & $\mathbf{3 1 7 1 . 9}$ & $\mathbf{3 5 8 1 . 6}$ & $\mathbf{4 1 3 2 . 1}$ & $\mathbf{5 0 5 8 . 9}$ & $\mathbf{6 4 4 3 . 2}$ & $\mathbf{8} \mathbf{0 6 9 . 6}$ & $\mathbf{1 1 0 8 2 . 0}$ & $\mathbf{8} \mathbf{9 7 5 . 0}$
\end{tabular}

\begin{tabular}{rrrrrrrrrrr}
55.1 & 80.6 & 37.3 & 16.8 & 16.8 & 31.2 & 97.8 & 84.9 & 100.3 & 49.8 & 14.8 \\
106.3 & 172.2 & 40.8 & 49.7 & 105.6 & 97.3 & 129.0 & 152.5 & 217.9 & 200.5 & 189.0 \\
53.3 & 235.6 & 237.6 & 62.6 & 241.5 & 426.6 & 489.5 & 363.3 & 731.8 & -739.2 & -368.2 \\
\hline $\mathbf{2 1 4 . 7}$ & $\mathbf{4 8 8 . 3}$ & $\mathbf{3 1 5 . 7}$ & $\mathbf{1 2 9 . 2}$ & $\mathbf{3 6 4 . 0}$ & $\mathbf{5 5 5 . 2}$ & $\mathbf{7 1 6 . 3}$ & $\mathbf{6 0 0 . 7}$ & $\mathbf{1 0 5 0 . 0}$ & $\mathbf{- 4 8 8 . 9}$ & $\mathbf{- 1 6 4 . 5}$ \\
& & & & & & & & & & \\
17.0 & 27.4 & 21.4 & 16.0 & 21.9 & 27.6 & 36.2 & 51.6 & 45.1 & 5.6 & 19.4 \\
32.2 & 32.4 & 36.1 & 33.3 & 32.9 & 38.7 & 47.6 & 57.6 & 66.8 & $\mathbf{7 4 . 5}$ & 60.2 \\
52.7 & 70.2 & 70.5 & 53.0 & 49.8 & 52.7 & 79.6 & 118.9 & 159.0 & 152.1 & 61.6 \\
\hline $\mathbf{1 0 2 . 0}$ & $\mathbf{1 3 0 . 1}$ & $\mathbf{1 2 8 . 1}$ & $\mathbf{1 0 2 . 3}$ & $\mathbf{1 0 4 . 6}$ & $\mathbf{1 1 9 . 0}$ & $\mathbf{1 6 3 . 3}$ & $\mathbf{2 2 8 . 1}$ & $\mathbf{2 7 0 . 9}$ & $\mathbf{2 3 2 . 2}$ & $\mathbf{1 4 1 . 2}$
\end{tabular}

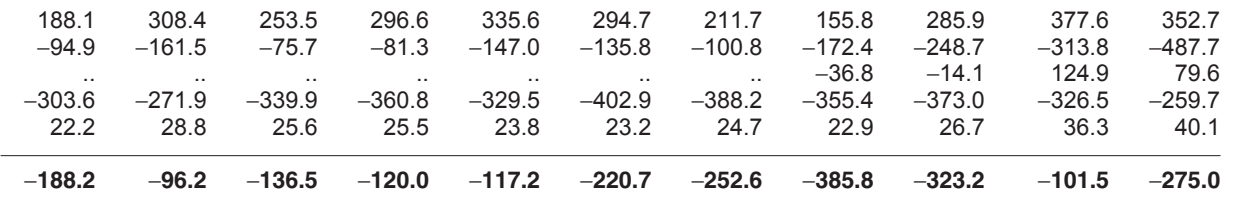

\begin{tabular}{rrrrrrrrrrr}
-70.5 & -75.0 & -5.5 & -18.3 & -24.1 & -20.3 & 53.8 & 39.9 & -62.3 & -37.8 & -15.4 \\
84.9 & 106.6 & -45.7 & 48.7 & 69.4 & -43.7 & -21.9 & 13.7 & 125.8 & 324.1 & 34.8 \\
2.7 & 1.6 & 8.4 & 1.0 & -5.4 & -7.9 & 9.6 & 20.6 & -27.0 & -121.7 & 29.1 \\
11.8 & -6.1 & 66.9 & -7.7 & -18.9 & 101.4 & -11.8 & -32.6 & -10.6 & -140.1 & -37.2 \\
0.6 & -3.9 & 3.1 & 0.5 & 1.6 & -0.2 & -0.7 & 0.4 & -1.2 & 1.3 & -5.8 \\
\hline 29.5 & $\mathbf{2 3 . 1}$ & $\mathbf{2 7 . 2}$ & $\mathbf{2 4 . 2}$ & $\mathbf{2 2 . 6}$ & $\mathbf{2} 29.4$ & $\mathbf{2 9 . 0}$ & $\mathbf{4 2 . 0}$ & $\mathbf{2 4 . 8}$ & $\mathbf{2 5 . 8}$ & $\mathbf{5 . 6}$
\end{tabular}

\begin{tabular}{rrrrrrrrrrr}
$\mathbf{2 9 . 5}$ & $\mathbf{2 3 . 1}$ & $\mathbf{2 7 . 2}$ & $\mathbf{2 4 . 2}$ & $\mathbf{2 2 . 6}$ & $\mathbf{2 9 . 4}$ & $\mathbf{2 9 . 0}$ & $\mathbf{4 2 . 0}$ & $\mathbf{2 4 . 8}$ & $\mathbf{2 5 . 8}$ & $\mathbf{5 . 6}$ \\
& & & & & & & & & & \\
16.1 & 17.6 & 25.3 & 35.5 & 33.2 & 35.7 & 43.0 & 32.0 & 46.3 & 61.7 & 53.7 \\
-6.4 & 0.5 & -1.2 & -0.8 & -0.4 & -2.0 & -2.2 & -2.4 & -0.7 & -6.9 & -5.7 \\
-12.2 & -17.3 & -15.7 & -17.2 & -16.1 & -16.0 & -19.0 & -20.7 & -25.5 & -26.8 & -16.9 \\
1.2 & 1.0 & 1.0 & 0.8 & 0.8 & 0.7 & 0.7 & 0.6 & 0.6 & 0.8 & 0.8 \\
\hline $\mathbf{- 1 . 2}$ & $\mathbf{1 . 8}$ & $\mathbf{9 . 4}$ & $\mathbf{1 8 . 2}$ & $\mathbf{1 7 . 5}$ & $\mathbf{1 8 . 3}$ & $\mathbf{2 2 . 5}$ & $\mathbf{9 . 4}$ & $\mathbf{2 0 . 8}$ & $\mathbf{2 8 . 8}$ & $\mathbf{3 2 . 0}$ \\
\hline
\end{tabular}

